\title{
Collaboration challenges faced by nurses when premature infants are discharged
}

Voie MP, Tunby J, Strømsvik N (2018) Collaboration challenges faced by nurses when premature infants are discharged. Nursing Children and Young People. 30, 2, 33-38. Date of submission: 2 I May 2017; date of acceptance: 4 January 2018. doi: I0.7748/ncyp.20I8.e960

\author{
Mona Pauline Voie \\ Neonatal intensive care \\ unit nurse, department of \\ pediatrics, division of child and \\ adolescent health, University \\ Hospital of North Norway, \\ Tromsø, Norway

\section{Jorunn Tunby division of child and adolescent health, University Hospital of North Norway, Tromsø, and associate professor, department of Artic Univeristy of Norway, Tromsø, Norway} \\ Research project coordinator, \\ health and care sciences, The

\section{Nina Strømsvik} \\ Head of familial cancer \\ center, department of medical \\ genetics, division of child and \\ adolescent health, University \\ Hospital of North Norway, \\ Tromsø, and associate \\ professor, department of \\ clinical medicine, The Arctic \\ University of Norway, Tromsø, \\ Norway
}

\section{Correspondence \\ nina@tbt.no}

\section{Peer review}

This article has been subject to open peer review and has been checked for plagiarism using automated software

\section{Conflict of interest}

This study was funded by the University Hospital of North Norway with one month free from work for the first author. The other authors declare no conflicts of interest

\begin{abstract}
Aim To explore the collaboration challenges faced by neonatal intensive care unit (NICU) nurses and public health nurses when preparing premature infants for transition home and to explore the culture of cooperation between the two professional groups.

Method Qualitative individual interviews were undertaken with two NICU nurses and two public health nurses. A qualitative content analysis was used to analyse the data.

Findings Three main themes were identified: different expectations and lack of communication between NICU nurses and public health nurses; responsibilities and interactions between the two professional groups were not clearly defined; and the public health nurses' competence was not recognised by the NICU nurses and parents.
\end{abstract}

Conclusion There is a need of clear guidelines about the discharge process from the NICU. This issue must be given further attention.

\section{Keywords}

babies, child health, neonatal, neonatal intensive care units, prematurity

\section{Introduction}

The parents of premature infants face challenges during neonatal intensive care unit (NICU) admissions and the transition home (Garfield et al 2014, Treyvaud et al 2014). Preparing parents for discharge through parent-infant interactions is the goal of healthcare providers (Als 2009, Landsem et al 2014). The transition from NICU to home and the importance of follow-up visits after discharge are being given increased attention (Purdy et al 2015). There has been less focus on the important role of the nurse in discharge planning, particularly cooperation between NICU nurses and public health nurses (Hintz et al 2008, Smith et al 2012, Jefferies 2014). Studies show that parents in NICU are not properly taught about discharge (Sneath 2009), and public health nurses have to take on this teaching instead (Newnam and Parrott 2013). Good teamwork and leadership are essential for effective discharge planning (Nosbusch et al 2011).

According to Østerlund (2008), only limited research has focused on the partnerships between different professions. The skills, perspectives and knowledge of other professionals should be acknowledged (Macdonald et al 2010, Petri 2010).
The need for improved cooperation between professions has been recognised, however, the implementation of changes to improve cooperation remains challenging (Kennedy and Stewart 2011). In Norway, where this pilot study took place, a health reform was announced in 2009 to improve interaction and communication between specialist services and primary healthcare workers (Helse- og omsorgsdepartementet 2009). The northern area of Norway occupies a large amount of land. Long travel distances, harsh weather conditions and a scattered population characterise this region. Only one university hospital in the region treats extreme premature infants.

\section{Aim}

The aim of this pilot study was to explore the collaboration challenges faced by NICU nurses and public health nurses when preparing premature infants for transition home and to explore the culture of cooperation between the two professional groups.

\section{Method}

Participants and procedures

Staff from the NICU sent invitation letters to several potential participants, which included information about the study. 


\author{
Acknowledgements \\ The authors would like to \\ thank the Division of Child \\ and Adolescent Health at the \\ University Hospital of North \\ Norway, the nurses who \\ participated in this study \\ and associate professor \\ Cathrine Arntsen at the Arctic \\ University of Norway
}

An open-ended question approach was used in interviews with two NICU nurses and two public health nurses. The interviews were recorded and fully transcribed by the first author. All participants were women with five to 15 years' experience. Three participants had more than ten years' experience. During the interviews three main areas were explored: the expectation about the meeting for planning transition home, communication between the two professional groups and future opportunities for their collaboration.

The interviews began with a question about expectations of a meeting between NICU nurses and public health nurses for planning transition home. The follow-up questions varied between interviews depending on participants' responses and they were also encouraged to expand on any other issues important to them.

\section{Data analysis}

Two people analysed the interviews using a qualitative content analysis. The transcripts were read twice to understand the context of each interview. Themes were decided on by identifying units of meaning related to the same central theme. The codes from every interview were colour-coded and assembled in a single document. Themes were generated based on differences, similarities and possibilities. In the last step of defining the concrete formulations of the themes, all three authors participated in the discussion (Graneheim and Lundman 2004, Braun and Clarke 2006).

\section{Ethical considerations}

Norwegian Science Data Services (grant number 25574) approved this study. All participants provided written consent. They were allowed to withdraw at any time. Audio files from the interviews were deleted on completion of the study. Quotes were modified to improve flow without changing any content.

\section{Findings}

Three main themes were identified:

»Different expectations and a lack of communication between NICU nurses and public health nurses.

» Responsibilities and interactions between the two groups of nurses were not clearly defined.

» Public health nurses' competence was not recognised by NICU nurses and parents.

Different expectations and a lack of communication between NICU nurses and public health nurses

The first theme described the lack of collaboration and communication when the two groups of nurses met in the NICU. It appeared to be difficult to put into words what happened when they met, as explained by a public health nurse:

'They are very positive... they are open to cooperation... it is not that they say: ... "No... we send only a summary... we do not have time to talk with you..." It is not like that...'

The NICU nurses expected the public health nurses to meet the parents, and explained:

'That is between them... Introducing them to each other and explaining that the new nurse the public health nurse the family will see. Therefore, it's up to the parents and public health nurse... that they are communicating.'

Both public health nurses confirmed this practice and expressed a need for the NICU nurses to participate in communicating with the parents. Spending time in the NICU entailed prioritising time, tasks and money, as well as the need to access information and expertise. NICU nurses noted that public health nurses often called and asked questions that the parents could have answered.

The public health nurses expressed a desire to acquire greater knowledge about the whole family's needs and challenges. The premature infants were referred to as 'breastfeeding and discharged' by the NICU nurses. Their summaries included limited information on each infant's birth and hospitalisation in the NICU.

NICU nurses thought it was important for public health nurses to experience the atmosphere of the NICU. The public health nurses recognised the importance of visiting the NICU; one described her experience:

'It was a very emotional and powerful experience... It made a strong impression on me... I became quite humble seeing these tiny, small children... and I thought about what a traumatic situation it would be to live in. Who are these parents coming home, and what kind of experience has this infant had? It was an incubator, a lot of stuff, equipment... what happens to the interaction...'

Responsibilities and interactions between the two groups of nurses were not clearly defined The second theme referred to the uncertainty about guidelines related to the transition home and the subsequent follow-up visits for the infant and family.

All participants were familiar with the professional guidelines for the follow-up of early born children (Markestad and Halvorsen 2007), where public health nurses 
are responsible for arranging the follow-up visits. The public health nurses expressed feelings of powerlessness and frustration about the lack of responsibility taken by the NICU nurses, and described them as being absent. The law about specialist responsibilities (Helse- og omsorgsdepartemente 1999), was referred to by one of the public health nurses as 'forgotten':

'It misses the part about the specialist's responsibility. It should be set... for example, what goes into a meeting?... When it's so vague...'

One of the public health nurses attempted to explain the limited communication and cooperation between the two groups:

'I think... that everyone is responsible for their own learning, that we rarely think that specialists are working inside hospitals. Primary healthcare service providers are not specialists. I do not know... if it is the result of everyone thinking that each of us is responsible for our own learning... ?'

NICU nurses explained their absence as a consequence of economic issues and recurring cutbacks in the NICU. They were also unsure who assumed responsibility for the infant's care:

'With the life that we live within the healthcare system today... it has to do with funding. Who should do what? Who will help these infants? What is the hospital's responsibility and what is the community's responsibility? It does not look to me that the staff from either the NICU or the hospital have the capability to follow-up... my experience is that the knowledge regarding follow-up visits is poor...unless the parents request it...'

The NICU nurses explained that their primary responsibility entailed the provision of lifesaving care and the supervision of the family in the hospital, and described cooperation with the public health nurses and the community services as: '... impractical to follow'.

Public health nurses' competence was not recognised by NICU nurses and parents The final theme described how NICU nurses did not recognise public health nurses' competence, which reduced them to passive listeners and limited their opportunity to add their expertise. Moreover, they believed that NICU nurses did not understand their role, nor did they understand the usefulness of other community services.

NICU nurses claimed that public health nurses lacked knowledge about premature infants and their unique healthcare needs.
Public health nurses regarded NICU nurses as hospital specialists and claimed to possess another expertise that enabled them to guide the family through the community healthcare system. A public health nurse described the meetings, telephone contact and discharge summaries written by NICU staff:

'Many times, I find that they have great ideas about what we can do when parents and infants get home... [laughs]. The fact is that they have added the list... They will need this and that... and that I have to deal with... Maybe we see it a different way... NICU nurses need to know more about what different community services can offer. If it is necessary in cases of extreme premature, I think both parents, the NICU nurses and the public health nurses shall meet and plan together. Maybe the NICU nurses could sit in with us... maybe that is not possible...'

One public health nurse expressed a desire to create a cooperative arena where they could exchange experiences and address relevant topics, but she received no feedback about this and felt 'betrayed by the specialists'.

Both NICU nurses regarded themselves as specialists dedicated to the treatment of premature infants and their special needs, and supporting parents during the difficult situation. Discharge planning and focusing on the public health nurses' need for information did not attract as much attention. One of the NICU nurses said:

'That is the basics... we want to be near the family... The most important thing is our closeness to both the parents and the infants. That we have the ability to provide cooperative care... that is the key for the parents...'

Public health nurses believed that most parents knew their children's medical needs when leaving the hospital but stated that they would require additional support as their infant grew. One public health nurse said: 'Many parents remember what they learned in the NICU. What has been said remains valid until confirmation [laughs]... We do not have anything to say because what was said in the NICU is still valid...'

Public health nurses expressed that they wanted to clarify the responsibility for further follow-up, but depended on support from the NICU nurses to make the family feel safe and comfortable in their care.

NICU nurses wanted public health nurses to have in mind an infant's prematurity when assessing their development. One NICU nurse described what she thought was important for the premature infant and the infant's family after the transition home:
Write for us

For information about writing for $\mathrm{RCNi}$ journals, contact writeforus@rcni.com

For author guidelines, go to rcni.com/ writeforus 
'We have knowledge that sometimes enables to predict what an infant and an infant's family need. That we can predict, or help them [public health nurse] to be aware of when to put in relief measures...It is important that someone fill this role...I think we shall convey not only what happened in the course, but also what we might expect of these measures in relation to age. And be aware that they may need special attention... and that parents need some interlocutors.'

\section{Discussion}

This pilot study involved a small sample of participants, and further research in larger populations is needed to increase reliability. However, the study may also apply to other disciplines in which collaboration between specialists and primary care services is important. This pilot study was undertaken in northern Norway. Health professions may differ between countries, but the collaboration challenges may be the same.

This pilot study revealed different expectations and a lack of communication between the NICU nurses and public health nurses about discharge planning for premature infants. The responsibilities and interactions between the two groups of nurses were not clearly defined. The study also identified a lack of recognition of the public health nurses' competence.

Our findings are consistent with those of previous studies that described a lack of discharge planning (Sneath 2009,

Nordhov et al 2010). This may result in rehospitalisation during the first two weeks after discharge, as well as within the first year of life (Houweling et al 2013). In this instance, empowering parents to deal with the situation is important. According to Antonovsky (1987) it is essential for parents to have control over their situation when coping with illness: comprehensibility and manageability are important. Providing parents with knowledge and support may enhance comprehensibility. Manageability refers to the ability to perceive available resources (Antonovsky 1987). It is essential that parents perceive NICU and public health nurses as available resources who may aid in their knowledge and expertise. Premature infants are vulnerable; they require primary care and specialist services more often than full-term infants (Wade et al 2008). To empower parents, the two groups of professionals need to collaborate.

The different goals of specialist and community care services mean that the different cultures have faced collaboration challenges (Helse- og omsorgsdepartementet 2009).
In terms of premature infant and family needs, a meeting between the NICU staff and primary healthcare services is strongly recommended to clarify responsibilities before the transition home (Markestad and Halvorsen 2007), but no specific guidelines for this meeting exist.

Despite the absence of directions this meeting was initiated by the public health nurses and NICU nurses. In northern Norway, the public health nurse is an important care provider for premature infants and their families, due to long distances, and public health nurses often work alone in small communities. Broedsgaard and Wagner (2005) described situations in which infants' homecomings were made easier for parents who had met the public health nurse before hospital discharge. This pilot study demonstrated, however, that NICU nurses did not recognise public health nurses' competence. Several studies have described the complexity in developing collaborative practice, where communication and clearly defined roles each represent criteria for success (Hall 2005, Suter et al 2009, Zwarenstein et al 2009). Insight into other providers' professional roles is also essential for developing collaborative practice. Lack of cooperation has a negative effect on patient care and services (Kvarnström 2008). This pilot study shows that these roles were not clearly defined. Important premises for enhancing communication between different professions are clarification of form, purpose, content and participants' roles (Østerlund 2008).

According to Hall (2005), professionals have had to define their own identities, as their values and spheres of practice are integral to establishing boundaries and developing and sustaining their authority. Suter et al (2009) observed that focusing on patients' needs will enable professionals to break down the barriers between them and their patients. In this case focusing on parents and the premature infant's needs after the discharge may improve collaboration between the professional groups.

In northern Norway, there is only one NICU that cares for extreme premature infants. When public health nurses visit this facility, they experience NICU nurses communicating in a space where medical and technological treatment dominate and where NICU nurses are the primary caregivers to the mother and infant.

Integrating human caring and relationship building within such confines may be difficult (Rice et al 2010). Nursing in this environment reflects a strong relationship between power, status and approval. 
Power sharing is one of the most complex aspects of interdisciplinary collaboration. The specialties, clinical territory and skills, may produce conflict when a nurse's competence is not acknowledged by the other nurse specialist (Rose 2011).

According to Foucault, power manifests itself in various elements of institutions, objects, feelings and ways of thinking. He attributed this to the relationship between power and knowledge, and how it creates a form of social control (Kelly 2013).

In light of Foucault's theory, an asymmetry in power is evident in this pilot study where communication between NICU nurses and public health nurses was one way. NICU nurses conveyed their knowledge and understanding through parents, through allowing public health nurses to see the incubator in the emergency room and by giving them brief information about the premature infant. This suggests that they chose to use the power they possessed in terms of knowledge, expertise and the benefit it gave them meeting in the NICU, which was a familiar and secure arena for them.

According to Freshwater et al (2014) and Reid et al (2008), higher education and professional work foster the development of identity and professional culture. Increased specialisation gives professionals less opportunities to interact with other disciplines (Hall and Weaver 2001), which may create a group that is more concerned about its own knowledge and culture. Findings in this pilot study may indicate different cultures between the two groups of nurses where strategies and attitudes were incorporated and maintained.

Guidelines exist for public health and NICU nurses about each group's knowledge and scope of practice (Markestad and Halvorsen, 2007). However, these guidelines appear to impede cooperation. Foucault (2003) described medical development and dominance, and how the need for specialised staff occurred. The training of NICU nurses providing specialised intensive care to infants started in Norway in 1971, with a focus on mastering the technical and medical challenges of caring for premature infants (Søjbjerg 2013).

This pilot study depicts a strong cultural and professional tradition of power that is rooted in history, but it also depicts recent changes in medicine. An increased focus on the individualised care of premature infants, their parents and the NICU nurses as supervisors of the infants' parents has taken hold (Als 2009, Gooding et al 2011).
In the emerging family-focused care model, the goal is to provide support for parents, enabling them to stimulate the parent-infant interactions necessary for optimal child development (Evans et al 2014).

However, it takes time to change long-held beliefs about nursing practices and medical decision-making (Freidson 1988, Lee et al 2014), which are dominated by power and a hierarchical structure (Adler et al 2008, Nugus et al 2010). This culture is manifested in the meetings between NICU and public health nurses. When public health nurses come to the NICU, their expectations are already set due to the overarching guidelines of Norwegian law (Helse- og omsorgsdepartemente 1999), which state that NICU nurses are specialists and providers of knowledge. The public health nurses who were interviewed confirmed these expectations.

Professionals' one-sided focus on themselves in education and practice is known. According to Hall (2005), the specialisation of knowledge has resulted in the specialisation of healthcare services with respect to professional groups. Healthcare services have struggled to define their territories, identities, values, spheres and roles in patient care (Hall 2005).

The specialisation of healthcare services has resulted in the development of a hierarchy in healthcare worldwide. The public health nurses in this study highlighted that mutual professional respect indirectly results in respect for parents and infants.

The need for post-discharge follow-up is well documented (Premji et al 2012). Raines (2013) described how mothers often do not disclose their concerns about their infant after discharge. The infant's prematurity and the parents' emotional vulnerability influence parental stress (Feeley et al 2011, Hynan et al 2013).

Having a premature infant is a traumatic experience that often persists long after discharge from the NICU, with feelings of guilt and disappointment (Smith et al 2012), and fear of what the future will bring. Rowe and Jones (2010) described the need to focus on infants' psychosocial health and for support not only during the acute phase of care.

An intervention study involving seven onehour counselling sessions in hospital and four home visits demonstrated significantly less stress among parents of premature infants (Landsem et al 2014). This pilot study shows parents' trust of NICU nurse competence. To benefit parents and infants, NICU nurses need to help parents understand the role of public health nurses (Garfield et al 2014).

\author{
Implications \\ for practice \\ "Collaboration \\ between NICU and \\ public health nurses \\ must improve and be \\ given priority to give \\ premature infants \\ and their families the \\ best chance to live \\ healthy lives \\ » Public health \\ nurses should be \\ more involved in \\ discharge planning \\ and establish a \\ relationship with the \\ family before they \\ leave hospital \\ »Discharge planning \\ requires better \\ guidelines and a \\ higher priority in \\ the NICU \\ » This pilot study \\ has identified \\ areas for future \\ research: teamwork, \\ communication \\ across boundaries \\ and parents' \\ perceptions \\ and expectations
}




\section{Online archive}

For related information, visit nursingchildren andyoungpeople. co.uk and search using the keywords

\section{Conclusion}

There is a lack of clear practices about the discharge process. Recommendations to ensure the best transition from the NICU to home for the premature infant, parents and family members must be given further attention.
Premature infants and their parents are vulnerable when leaving the hospital. The two groups of professionals, NICU nurses and public health nurses, need to collaborate to provide best knowledge and expertise for the family.

\section{References}

Adler PS, Kwon S-W, Heckscher C (2008)

Perspective-professional work: the emergence of collaborative community. Organization Science.

$19,2,359-376$.

Als H (2009) Newborn Individualized Developmental Care and Assessment Program (NIDCAP): new frontier for neonatal and perinatal medicine. Journal of Neonatal-Perinatal Medicine 2 3, 135-147.

Antonovsky A (1987) Unraveling the Mystery of Health: How People Manage Stress and Stay Well. Jossey-Bass, San Francisco CA.

Braun V, Clarke V (2006) Using thematic analysis in psychology. Qualitative Research in Psychology. $3,2,77-101$.

Broedsgaard A, Wagner L (2005) How to facilitate parents and their premature infant for the transitio home. International Nursing Review. 52, 3, 196-203.

Evans T, Whittingham K, Sanders M et al (2014) Are parenting interventions effective in improving the relationship between mothers and their preterm infants? Infant Behaviour and Development. 37, 2, 131-154.

Feeley N, Zelkowitz P, Cormier C et al (2011) Posttraumatic stress among mothers of very low birthweight infants at 6 months after discharge from the neonatal intensive care unit. Applied Nursing Research. 24, 2, 114-117

Foucault M (2003) The Birth of the Clinic: An Archaeology of Medical Perception. Routledge, Abingdon.

Freidson E (1988) Profession of Medicine: A Study of the Sociology of Applied Knowledge. University of Chicago Press, Chicago IL.

Freshwater D, Cahill J, Essen C (2014) Discourses of collaborative failure: identity, role and discourse in an interdisciplinary world. Nursing Inquiry. $21,1,59-68$

Garfield CF, Lee Y, Kim HN (2014) Paternal and maternal concerns for their very low-birth-weight infants transitioning from the NICU to home. Journ of Perinatal and Neonatal Nursing. 28, 4, 305-312.

Gooding JS, Cooper LG, Blaine Al et al (2011) Family support and family-centered care in the neonat intensive care unit: origins, advances, impact. Seminars in Perinatology. 35, 1, 20-28

Graneheim UH, Lundman B (2004) Qualitative content analysis in nursing research: concepts, procedures and measures to achieve trustworthiness. Nurse Education Today. $24,2,105-112$
Hall P (2005) Interprofessional teamwork: professional cultures as barriers. Journal of Interprofessional Care. 19, Suppl 1, 188-196.

Hall P, Weaver L (2001) Interdisciplinary education and teamwork: a long and winding road. Medica Education. 35, 9, 867-875.

Helse- og omsorgsdepartemente (1999) LOV1999-07-02 nr 61: lov om spesialisthelsetjenesten m.m. (spesialisthelsetjenesteloven). www. lovdata.no/dokument/NL/lov/1999-07-02-6 (Last accessed: 25 lanuary 2018.

Helse- og omsorgsdepartementet (2009) Samhandlingsreformen: Rett behandling - på rett sted - til rett tid. St.meld. nr. 47 (2008-2009). Regjeringen 149 , 0 slo.

Hintz SR, Kendrick DE, Vohr BR et al (2008) Community supports after surviving extremely low-birth-weight, extremely preterm birth: special outpatient services in early childhood. Archives of Pediatrics and Adolescent Medicine. 162, 8, 748-755.

Houweling LM, Bezemer ID, Penning-van Beest FJ et al (2013) First year of life medication use and hospital admission rates: premature compared with term infants. Journal of Pediatrics. 163, 1, 61-6. e1.

Hynan M, Mounts K, Vanderbilt DL (2013) Screening parents of high-risk infants for emotional distress: rationale and recommendations. Journal of Perinatology. 33, 10,748.

Jefferies AL (2014) Going home: facilitating discharge of the preterm infant. Paediatrics and Child Health. 19, 1,31-42

Kelly MGE (2013) Foucault's History of Sexuality Volume 1, The Will to Knowledge. Edinburgh University Press, Edinburgh.

Kennedy S, Stewart H (2011) Collaboration between occupational therapists and teachers: definitions, implementation and efficacy. Australian Occupational Therapy Journal. 58, 3, 209-214.

Kvarnström S (2008) Difficulties in collaboration: a critical incident study of interprofession healthcare teamwork. Journal of Interprofessional Care. 22, 2, 191-203.

Landsem IP, Handegård BH, Tunby J et al (2014) Early intervention program reduces stress in parents of preterms during childhood, a randomized controlled trial. Trials. 15, 387

Lee LA, Carter M, Stevenson SB et al (2014) Improving family-centered care practices in the NICU. Neonatal Network. 33, 3, 125-132.
Macdonald MB, Bally JM, Ferguson LM et al (2010) Knowledge of the professional role of others: a key interprofessional competency. Nurse Education in Practice. 10, 4, 238-242.

Markestad T, Halvorsen B (2007) Faglige retningslinjer for oppfølging av for tidlig fødte barn. Sosial- og helsedirektoratet, Oslo.

Newnam K, Parrott J (2013) The NICU graduate: implications for pediatric primary care. Newborn and Infant Nursing Reviews. 13, 2, 94-100.

Nordhov SM, Kaaresen PI, Rønning JA et al (2010) A randomized study of the impact of a sensitizing intervention on the child-rearing attitudes of parents of low birth weight preterm infants. Scandinavian Journal of Psychology. 51, 5, 385-391.

Nosbusch JM, Weiss ME, Bobay KL (2011) An integrated review of the literature on challenges confronting the acute care staff nurse in discharge planning. Journal of Clinical Nursing 20,754-774.

Nugus P, Greenfield D, Travaglia J et al (2010) How and where clinicians exercise power: interprofessional relations in health care. Social Science and Medicine. 71, 5, 898-909.

sterlund C (2008) The materiality of communicative practices. Scandinavian Journal of Information Systems. 20, 1, 7-40

Purdy I, Craig J,Zeanah P (2015) NICU discharge planning and beyond: recommendations for parent psychosocial support. Journal of Perinatology. 35, S24-8.

Pethybridge J (2004) How team working influences discharge planning from hospital: a study of four multi-disciplinary teams in an acute hospital in England. Journal of Interprofessional Care. $18,1,29-41$.

Petri L (2010) Concept analysis of interdisciplinary collaboration. Nursing Forum. 45, 2, 73-82.

Premii SS, Young M, Rogers C et al (2012) Transitions in the early-life of late preterm infants vulnerabilities and implications for postpartum care. Journal of Perinatal and Neonatal Nursing $26,1,57-68$.

Raines DA (2013) Preparing for NICU discharge: mothers' concerns. Neonatal Network.

$32,6,399-403$

Reid A, Dahlgren L0, Petocz P et al (2008) Identity and engagement for professional formation. Studies in Higher Education. 33, 6, 729-742.
Rice K, Zwarenstein M, Conn LG etal (2010) An intervention to improve interprofessioan collaboration and communications: a comparative qualitive study. Journal of Interprofessional Care 24, 4,350-261.

Rose L (2011) Interprofessional collaboration in the ICU: how to define? Nursing in Critical Care. 61, 5-10. I will need to delete this if the reference cited in the text isn't needed.

Rowe J, Jones L (2010) Discharge and beyond. A ongitudinal study comparing stress and coping in parents of preterm infants. Journal of Neonatal Nursing. 16, 6, 258-266.

Smith VC, Dukhovny D, Zupancic JA et al (2012) Neonatal intensive care unit discharge preparedness: primary care implications. Clinica Pediatrics. 51, 5, 454-461

Sneath N (2009) Discharge teaching in the NICU: are parents prepared? An integrative review of parents' perceptions. Neonatal Network. 28, 4, 237-246.

Søjbjerg I (2013) Hvorfor barnesykepleiere? Tidsskrift for Barnesykepleiere. 17,11-12

Suter E, Arndt J, Arthur N et al (2009) Role understanding and effective communication as core competencies for collaborative practice. Journal of Interprofessional Care. 23, 1, 41-51.

Treyvaud K, Lee KJ, Doyle LW et al (2014) Very preterm birth influences parental mental health and family outcomes seven years after birth. Journal of Pediatrics. 164, 3, 515-521.

Wade KC, Lorch SA, Bakewell-Sachs S et al 2008) Pediatric care for preterm infants after NICU discharge: high number of office visits and prescription medications. Journal of Perinatology. $28,10,696-701$

Zwarenstein M, Goldman J, Reeves S (2009) Interprofessional collaboration: effects of practicebased interventions on professional practice and healthcare outcomes. Cochrane Database of Systematic Reviews. Issue 3. CD000072. 